\title{
New Rough Approximations Based on E-Neighborhoods
}

\author{
Tareq M. Al-shami $\mathbb{D}^{1,2}$ Wen Qing Fu $\mathbb{D}^{1}{ }^{1}$ and E. A. Abo-Tabl ${ }^{3,4}$ \\ ${ }^{1}$ Freshman Academy, Xi'an Technological University, Xi'an 710021, China \\ ${ }^{2}$ Department of Mathematics, Sana'a University, Sana'a, Yemen \\ ${ }^{3}$ Department of Mathematics, College of Arts and Science, Methnab, Qassim University, Buridah, Saudi Arabia \\ ${ }^{4}$ Department of Mathematics, Faculty of Science, Assiut University, Assiut, Egypt \\ Correspondence should be addressed to Wen Qing Fu; palace_2000@163.com
}

Received 7 December 2020; Revised 15 January 2021; Accepted 30 January 2021; Published 2 March 2021

Academic Editor: Abdel-Haleem Abdel-Aty

Copyright (c) 2021 Tareq M. Al-shami et al. This is an open access article distributed under the Creative Commons Attribution License, which permits unrestricted use, distribution, and reproduction in any medium, provided the original work is properly cited.

\begin{abstract}
This paper puts forward some rough approximations which are motivated from topology. Given a subset $R \subseteq U \times U$, we can use 8 types of $E$-neighborhoods to construct approximations of an arbitrary $X \subseteq U$ on the one hand. On the other hand, we can also construct approximations relying on a topology which is induced by an $E$-neighborhood. Properties of these approximations and relationships between them are studied. For convenience of use, we also give some useful and easy-to-understand examples and make a comparison between our approximations and those in the published literature.
\end{abstract}

\section{Introduction and Preliminaries}

The problem of imperfect knowledge became a crucial issue for computer scientists, especially in the area of information system and artificial intelligence $[1,2]$. There are various approaches to manipulate and understand imperfect knowledge, among which is rough set theory. Rough set was proposed by Pawlak [3, 4] in 1982 which has been generalized in many ways [5-13]. What we are concerned about are those methods whose ideas are motivated from topology, for example, methods constructing the lower and upper approximations by using different kinds of neighborhoods, such as right and left neighborhoods $[10,14]$, minimal right neighborhoods [15], and intersection and union neighborhoods (see $[16,17])$. In fact, a combination of rough set theory and topological theory became the main goal of many studies (see [18-25]).

The present paper is a continuation to these works where we initiate new types of neighborhoods (namely, $E_{j}$-neighborhoods) to give lower and upper approximations of an arbitrary set $X$ directly or indirectly. In Section 2, we apply $N_{j}$-neighborhoods to establish the concepts of $E_{j}$-neighborhoods and discuss the main properties. With the help of examples, we show the relationships between them as well as with $N_{j}$-neighborhoods and $P_{j}$-adhesion neighborhoods. In Section 3, we formulate and study the concepts of $E_{j}$-lower and $E_{j}$-upper approximations, $E_{j}$-boundary region, $E_{j}$-positive and $E_{j}$-negative regions, and $E_{j}$-accuracy measure of a subset and make comparisons between them with respect to different types of $j$. In Section 4 , we study the previous concepts from a topological view and explore their main properties. In Section 5, we give some conclusions and make a plan for future works.

Now, we recall some basic properties and results of rough set theory, particularly those related to some types of neighborhood systems.

Definition 1 (see $[3,5,16])$

(1) A subset $R \subseteq U \times U$ (also called a binary relation on $U$ ) is said to be an equivalence relation if it is reflexive (i.e., $(v, v) \in R$ for each $v \in U)$, symmetric (i.e., $(u, v) \in R$ if $(v, u) \in R)$, and transitive (i.e., $(u, w) \in R$ whenever $(u, v) \in R$ and $(v, w) \in R)$. It is said to be a preorder (or quasi-order) if it is reflexive and transitive. It is said to be a partial order if it is an antisymmetric (i.e., $u=v$ whenever $(u, v) \in R$ and 
$(v, u) \in R)$ preorder. It is said to be a diagonal if $R=\{(v, v): v \in U\}$. It said to be serial if for every $v \in U, w \in U$ such that $(v, w) \in R$ (also written as $v R w)$.

(2) (See $[4,26]$ for a special case). For an equivalence relation $R$ on $U$ and a subset $X \subseteq U$, the two related sets $\underline{R}(X)=\bigcup\{A \in U / R: A \subseteq X\}$ and $\underline{R}(X)=\bigcup$ $\{A \in U / R: A \subseteq X \neq \varnothing\}$ are called the Pawlak lower approximation and upper approximation of $X$, respectively (where $U / R=\{[x]: x \in U\}$ is the set of all equivalence classes).

Proposition 1 (see $[3,4]$ for a special case). The lower approximations and the upper approximations have the following properties $\left(\{E, F\} \cup\left\{E_{i}\right\}_{i \in I} \subseteq 2^{U}\right.$, the power set of $\left.U\right)$ :

(1) $\underline{R}(\varnothing)=\bar{R}(\varnothing)=\varnothing$ and $\underline{R}(U)=\bar{R}(U)=U$.

(2) $\underline{R}(E) \subseteq E \subseteq \bar{R}(E)$ and $\underline{R}(E)=\left[\bar{R}\left(E^{c}\right)\right]^{c}$.

(3) $\underline{R}\left(\cup_{i \in I} E_{i}\right)=\cup_{i \in I} \underline{R}\left(E_{i}\right)$ and $\underline{R}\left(\cup_{i \in I} E_{i}\right)=\cup_{i \in I}$ $\underline{R}\left(E_{i}\right)$. Particularly, $\underline{R}(E) \subseteq \underline{R}(F)$ and $\underline{R}(E) \subseteq \underline{R}(F)$ if $E \subseteq F$.

(4) $\underline{R}[\underline{R}(E)]=\underline{R}(E)$ and $\bar{R}[\bar{R}(E)]=\bar{R}(E)$.

Definition 2. A subset $\mathscr{J} \subseteq 2^{U}$ is called a topology on $U$ (and $(U, \mathscr{F})$ is called a topological space) if it is closed under arbitrary union and finite intersection. A topology satisfying that every open set is also closed is called a clopen topology. We will use $A^{o}$ to denote the interior of $A$ (i.e., the union of all open sets that are contained in $A$ ) and $A^{-}$to denote the closure of $A$ (i.e., the intersection of all closed sets containing $A)$ in this paper.

Let $\mathscr{J}_{R}=\left\{A \in 2^{U}:[x] \subseteq A(\forall x \in A)\right\}$. Then, $\left(U, \mathscr{J}_{R}\right)$ is a topological space, $R(X)=X^{o}, \bar{R}(X)=X^{-}$, and $[x]$ is the smallest open neighborhood of $x(\forall x \in U)$. This inspires many people to define the lower approximation and the upper approximation by neighborhoods; actually, our approach in this paper is also motivated from topology. In the following, we write $J=\{r, l,\langle r\rangle,\langle l\rangle, i, u,\langle i\rangle,\langle u\rangle\}$.

Definition 3 (see $[10,14,16]$ ). Let $R \subseteq U^{2}$ and $j \in J$.

(1) The 8 kinds of $j$-neighborhoods are defined as follows: $\quad N_{r}(v)=\{w \in U: v R w\}, \quad N_{l}(v)=\{w \in U$ : $w R v\}, \quad N_{\langle r\rangle}(v)=\cap\left\{N_{r}\right\}(w): v \in N_{r}(w) \quad$ if $v \in N_{r}(w)$ for some $w \in U$ or $\varnothing$, otherwise), $N_{\langle l\rangle}(v)=\cap\left\{N_{l}\right\}(w): v \in N_{l}(w)$ (if $v \in N_{l}(w)$ for some $w \in U$ or $\varnothing$, otherwise), $N_{i}(v)=N_{r}(v) \cap$ $N_{l}(v), N_{u}(v)=N_{r}(v) \cup N_{l}(v), N_{\langle i\rangle}(v)=N_{\langle r\rangle}(v) \cap$ $N_{\langle l\rangle}(v)$, and $N_{\langle u\rangle}(v)=N_{\langle r\rangle}(v) \cup N_{\langle l\rangle}(v)$

(2) The triple $\left(U, R, \lambda_{j}\right)$ is called a $j$-neighborhood space (in brief, $j$-NS), where $\lambda_{j}$ is a mapping from $U$ to $2^{U}$ which associated each $v \in U$ with a $j$-neighborhood

Definition 4 (see [6]). Let $R \subseteq U^{2}$. The $j$-adhesion neighborhoods are defined as follows:
(1) $P_{r}(v)=\left\{w \in U: N_{r}(w)=N_{r}(v)\right\}$

(2) $P_{l}(v)=\left\{w \in U: N_{l}(w)=N_{l}(v)\right\}$

(3) $P_{\langle r\rangle}(v)=\left\{w \in U: N_{\langle r\rangle}(w)=N_{\langle r\rangle}(v)\right\}$

(4) $P_{\langle l\rangle}(v)=\left\{w \in U: N_{\langle l\rangle}(w)=N_{\langle l\rangle}(v)\right\}$

(5) $P_{i}(x)=P_{r}(v) \cap P_{l}(v)$

(6) $P_{u}(x)=P_{r}(v) \cup P_{l}(v)$

(7) $P_{\langle i\rangle}(v)=P_{\langle r\rangle}(v) \cap P_{\langle l\rangle}(v)$

(8) $P_{\langle u\rangle}(v)=P_{\langle r\rangle}(v) \cup P_{\langle l\rangle}(v)$

\section{E-Neighborhoods}

In this section, we introduce the notions of $E$-neighborhoods using $j$-neighborhoods and study their properties.

Definition 5. Let $R \subseteq U^{2}$. The E-neighborhoods are defined as follows:

(1) $E_{r}(x)=\left\{y \in U: N_{r}(y) \cap N_{r}(x) \neq \varnothing\right\}$

(2) $E_{l}(x)=\left\{y \in U: N_{l}(y) \cap N_{l}(x) \neq \varnothing\right\}$

(3) $E_{i}(x)=E_{r}(x) \cap E_{l}(x)$

(4) $E_{u}(x)=E_{r}(x) \cup E_{l}(x)$

(5) $E_{\langle r\rangle}(x)=\left\{y \in U: N_{\langle r\rangle}(y) \cap N_{\langle r\rangle}(x) \neq \varnothing\right\}$

(6) $E_{\langle l\rangle}(x)=\left\{y \in U: N_{\langle l\rangle}(y) \cap N_{\langle l\rangle}(x) \neq \varnothing\right\}$

(7) $E_{\langle i\rangle}(x)=E_{\langle r\rangle}(x) \cap E_{\langle l\rangle}(x)$

(8) $E_{\langle u\rangle}(x)=E_{\langle r\rangle}(x) \cup E_{\langle l\rangle}(x)$

We give the following example to illustrate how we calculate different types of neighborhoods. Also, we will benefit from this example to clarify some obtained results.

Example 1. Let $U=\{v, w, x, y\}$ and $R=\{(v, v),(y, y)$, $(v, x),(v, y),(y, w),(w, y)\}$. Then, the $j$-neighborhoods and $E$-neighborhoods of a point are as in Table 1.

Theorem 1. E-neighborhoods have the following properties $\left(R \subseteq U^{2}, v \in U\right)$ :

(1) $E_{i}(v) \subseteq E_{r}(v) \cap E_{l}(v) \subseteq E_{r}(v) \cup E_{l}(v) \subseteq E_{u}(v)$

(2) $E_{\langle i\rangle}(v) \subseteq E_{\langle r\rangle}(v) \cap E_{\langle l\rangle}(v) \subseteq E_{\langle r\rangle}(v) \cup E_{\langle l\rangle}(v) \subseteq$ $E_{\langle u\rangle}(v)$

(3) $v \in E_{j}(x)$ iff $x \in E_{j}(v)(j \in J)$

(4) If $R$ is reflexive, then $E_{\langle j\rangle}(v) \subseteq E_{j}(v)$ and $P_{j}(v) \cup N_{j}(v) \subseteq E_{j}(v)(j \in J)$

(5) If $R$ is symmetric, then $E_{r}(v)=E_{l}(v)=E_{i}(v)=$ $E_{u}(v)$ and $E_{\langle r\rangle}(x)=E_{\langle l\rangle}(v)=E_{\langle i\rangle}(v)=E_{\langle u\rangle}(v)$

(6) If $R$ is transitive, then $E_{j}(v) \subseteq E_{\langle j\rangle}(v)(j \in\{r, l u, i\})$

(7) If $R$ is serial, then $P_{j}(v) \subseteq E_{j}(v)(j \in J)$

(8) If $R$ is symmetric and transitive, then $E_{j}(v) \subseteq N_{j}(v)$ and $E_{j}(v) \subseteq E_{j}(w)$ (if $\left.v \in E_{j}(w)\right)$ for each $j \in J$

(9) If $R$ is preorder, then $E_{j}(v) \subseteq E_{\langle j\rangle}(v)(j \in\{r, l, u, i\})$

(10) If $R$ is a equivalence relation, then for each $j \in J$, all $E_{j}(v)$ are identical, $E_{j}(v)=N_{j}(v)=P_{j}(v)$, and $v \in E_{j}(w)$ iff $E_{j}(v)=E_{j}(w)$ 
TABLE 1: $j$-neighborhoods and $E$-neighborhoods.

\begin{tabular}{lcccc}
\hline & $v$ & $w$ & $x$ & $y$ \\
\hline$N_{r}$ & $\{v, x, y\}$ & $\{y\}$ & $\varnothing$ & $\{w, y\}$ \\
$N_{l}$ & $\{v\}$ & $\{y\}$ & $\{v\}$ & $\{v, w, y\}$ \\
$N_{i}$ & $\{v\}$ & $\{y\}$ & $\varnothing$ & $\{w, y\}$ \\
$N_{u}$ & $\{v, x, y\}$ & $\{y\}$ & $\{v\}$ & $\{v, w, y\}$ \\
$N_{\langle r\rangle}$ & $\{v, x, y\}$ & $\{w, y\}$ & $\{v, x, y\}$ & $\{y\}$ \\
$N_{\langle l\rangle}$ & $\{v\}$ & $\{v, w, y\}$ & $\varnothing$ & $\{y\}$ \\
$N_{\langle i\rangle}$ & $\{v\}$ & $\{w, y\}$ & $\varnothing$ & $\{y\}$ \\
$N_{\langle u\rangle}$ & $\{v, x, y\}$ & $\{v, w, y\}$ & $\{v, x, y\}$ & $\{y\}$ \\
$P_{r}$ & $\{v\}$ & $\{w\}$ & $\{x\}$ & $\{y\}$ \\
$P_{l}$ & $\{v, x\}$ & $\{w\}$ & $\{v, x\}$ & $\{y\}$ \\
$P_{i}$ & $\{v\}$ & $\{w\}$ & $\{x\}$ & $\{y\}$ \\
$P_{u}$ & $\{v, x\}$ & $\{w\}$ & $\{v, x\}$ & $\{y\}$ \\
$P_{\langle r\rangle}$ & $\{v, x\}$ & $\{w\}$ & $\{v, x\}$ & $\{y\}$ \\
$P_{\langle l\rangle}$ & $\{v\}$ & $\{w\}$ & $\{x\}$ & $\{y\}$ \\
$P_{\langle i\rangle}$ & $\{v\}$ & $\{w\}$ & $\{x\}$ & $\{y\}$ \\
$P_{\langle u\rangle}$ & $\{v, x\}$ & $\{w\}$ & $\{v, x\}$ & $\{y\}$ \\
$E_{r}$ & $\{v, w, y\}$ & $\{v, w, y\}$ & $\varnothing$ & $\{v, w, y\}$ \\
$E_{l}$ & $\{v, x, y\}$ & $\{w, y\}$ & $\{v, x, y\}$ & $U$ \\
$E_{i}$ & $\{v, y\}$ & $\{w, y\}$ & $\varnothing$ & $\{v, w, y\}$ \\
$E_{u}$ & $U$ & $\{v, w, y\}$ & $\{v, x, y\}$ & $U$ \\
$E_{\langle r\rangle}$ & $U$ & $U$ & $U$ & $U$ \\
$E_{\langle l\rangle}$ & $\{v, w\}$ & $\{v, w, y\}$ & $\varnothing$ & $\{w, y\}$ \\
$E_{\langle i\rangle}$ & $\{v, w\}$ & $\{v, w, y\}$ & $\varnothing$ & $\{w, y\}$ \\
$E_{\langle u\rangle}$ & $U$ & $U$ & $U$ & $U$ \\
\hline & & & &
\end{tabular}

Proof. (3) Obviously, $\quad v \in E_{j}(x) \Leftrightarrow N_{j}(v) \cap N_{j}(x) \neq \varnothing \Leftrightarrow$ $x \in E_{j}(v)$ for each $j \in\{r, l,\langle r\rangle,\langle l\rangle\}$. Then, $v \in E_{j}(x) \Leftrightarrow x \in$ $E_{j}(v)$ for each $j \in\{i, u,\langle i\rangle,\langle u\rangle\}$.

(4) Step 1: since $R$ is reflexive, $\cap_{v \in N_{r}(x)} N_{r}(x) \subseteq N_{r}(v)$ and $\cap_{v \in N_{l}(x)} N_{l}(x) \subseteq N_{l}(v)$. This implies that $E_{\langle r\rangle}(v) \subseteq E_{r}(v)$ and $E_{\langle l\rangle}(v) \subseteq E_{l}(v)$. Consequently, $E_{\langle i\rangle}(v) \subseteq E_{i}(v)$ and $E_{\langle u\rangle}(v) \subseteq E_{u}(v)$.

Step 2: let $x \in N_{j}(v)$. By reflexivity of $R$, we have $\{x\} \subseteq N_{j}(x) \cap N_{j}(v)$. Therefore, $x \in E_{j}(v)$; thus, $N_{j}(v) \subseteq E_{j}(v)$. Also, let $x \in P_{j}(v)$. Then, $N_{j}(x)=N_{j}$ $(v)$. Since $R$ is reflexive, $N_{j}(x) \cap N_{j}(v) \neq \varnothing$. Therefore, $x \in E_{j}(v)$; thus, $P_{j}(v) \subseteq E_{j}(v)$. Hence, we obtain the desired result.

(5) Since $R$ is symmetric, $N_{r}(v)=N_{l}(v)$. Therefore, $N_{r}(v) \cap N_{r}(z) \neq \varnothing \Leftrightarrow N_{l}(v) \cap N_{l}(z) \neq \varnothing$; thus, $E_{r}$ $(v)=E_{l}(v)$. Consequently, $E_{r}(v)=E_{l}(x)=E_{i}(x)=$ $E_{u}(x)$. Similarly, $\quad E_{\langle r\rangle}(x)=E_{\langle l\rangle}(v)=E_{\langle i\rangle}(v)=$ $E_{\langle u\rangle}(v)$.

(6) Let $a \in N_{r}(v)$. Then, $v R a$. For each $N_{r}(x)$ containing $v$, we have $x R v$. Since $R$ is transitive, $x R a$. Therefore, $a \in N_{r}(x)$; thus, $a \in \cap_{v \in N_{r}(x)} N_{r}(x)=$ $N_{\langle r\rangle}(v)$. Hence, $N_{r}(v) \subseteq N_{\langle r\rangle}(v)$; consequently, $E_{r}(v) \subseteq E_{\langle r\rangle}(v)$. Similarly, $E_{l}(v) \subseteq E_{\langle l\rangle}(v)$. This implies that $E_{i}(v) \subseteq E_{\langle i\rangle}(v)$ and $E_{u}(v) \subseteq E_{\langle u\rangle}(v)$.

(7) It follows from (4) and the fact $v \in E_{j}(x) \Leftrightarrow N_{j}$ $(v) \cap N_{j}(x) \neq \varnothing \Leftrightarrow x \in E_{j}(v)(j \in\{r, l,\langle r\rangle,\langle l\rangle\})$.

(8) Step 1: we prove the case $j=r$. Let $x \in E_{r}(v)$. Then,
$N_{r}(x) \cap N_{r}(v) \neq \varnothing$, i.e., there exists $z \in N_{r}(x) \cap$ $N_{r}(v), x R z$, and $v R z$. Since $R$ is symmetric and transitive, $\quad v R x$. Therefore, $\quad x \in N_{r}(v)$; thus, $E_{r}(v) \subseteq N_{r}(v)$.

Step 2: since $R$ is symmetric, $E_{r}(v)=E_{l}(v)=E_{i}(v)=$ $E_{u}(v)$ by (5). We only prove the case $j=l$. Let $v \in E_{l}(w)$. Then, $N_{l}(v) \cap N_{l}(w) \neq \varnothing$; therefore, there is $a \in U$ such that $a R v$ and $a R w$. Now, let $x \in E_{l}(v)$. Then, $N_{l}(x) \cap N_{l}(v) \neq \varnothing$, i.e., there exists a $b \in U$ such that $b R x$ and $b R v$. Note that $a R b$; this means that $a R x$; consequently, $a \in N_{l}(x)$; also, $a \in N_{l}(w)$; thus, $N_{l}(x) \cap N_{l}(x) \neq \varnothing$. Hence, $x \in E_{l}(w)$, as required.

(10) Step 1: by (4) and (8), $E_{j}(v)=N_{j}(v)$ and $P_{j}(v) \subseteq E_{j}(v)$. It remains to prove $E_{j}(v) \subseteq P_{j}(v)$. Since $R$ is symmetric, $E_{r}(v)=E_{l}(v)=E_{i}(v)=E_{u}(v)$ by $(5)$. Let $x \in E_{l}(v)$. Then, $N_{l}(x) \cap N_{l}(v) \neq \varnothing$. Since $R$ is equivalence, $N_{l}(x)=N_{l}(v)$. Therefore, $x \in P_{l}(v)$; consequently, $E_{l}(v) \subseteq P_{l}(v)$. Thus, $P_{j}(v)=E_{j}(v)$ is proved.

Step 2: we only prove the case $j=l$. Let $v \in E_{l}(w)$. Then, $N_{l}(v) \cap N_{l}(w) \neq \varnothing$. Since $R$ is equivalence, $N_{l}(v)=N_{l}(w) \neq \varnothing$. Therefore, $E_{j}(v)=E_{j}(w)$. Conversely, $E_{j}(v)=E_{j}(w)$. Since $R$ is reflexive, $v \in N_{l}(v)$. Hence, $v \in E_{j}(w)$.

\section{Rough Approximations Using E-Neighborhoods Directly}

Let $\left(U, R, \lambda_{j}\right)$ be $j$-NS and $\mathscr{R}_{j}=\left\{E_{j}(v): v \in U\right\}(j \in J)$. We devote this section to formulate the following concepts using E-neighborhoods directly: $\mathscr{R}_{j}$-lower approximation, $\mathscr{R}_{j}$-upper approximation, $\mathscr{R}_{j}$-boundary region, $\mathscr{R}_{j}$-positive region, $\mathscr{R}_{j}$-negative region, and $\mathscr{R}_{j}$-accuracy measure of a subset $X$. We will also illustrate the relationships between them and reveal the main properties with the help of examples.

Definition 6. Let $X \subseteq U$ and $j \in J$. Then, $\mathscr{R}_{j}^{-}(X)=\left\{x \in U: E_{j}(x) \subseteq X\right\} \quad$ and $\quad \mathscr{R}_{j}^{+}(X)=\{x \in U$ : $\left.E_{j}(x) \subseteq X \neq \varnothing\right\}$ are called $\mathscr{R}_{j}$-lower approximation and $\mathscr{R}_{j}$-upper approximation, respectively; $\mathscr{B}_{j}(X)=\mathscr{R}_{j}^{+}(X)-$ $\mathscr{R}_{j}^{-}(X), \operatorname{POS}_{j}(X)=\mathscr{R}_{j}^{-}(X)$, and $\mathrm{NEG}_{j}(X)=U-\mathscr{R}_{j}^{+}(X)$ are called $\mathscr{R}_{j}$-boundary, $\mathscr{R}_{j}$-positive, and $\mathscr{R}_{j}$-negative regions of $X$, respectively; $\mathscr{M}_{j}(X)=\left(\left|\mathscr{R}_{j}^{-}(X) \cap X\right| / \mid \mathscr{R}_{j}^{+}\right.$ $(X) \cap X \mid)$ is called the $\mathscr{R}_{j}$-accuracy measure of $X$ (if $\left.0<\left|\mathscr{R}_{j}^{+}(X) \cup X\right|<\aleph_{0}\right)$, where $|X|$ denotes the cardinality of a set $X$ and $\aleph_{0}$ denotes the cardinality of the set of natural numbers.

Theorem 2. $\mathscr{R}_{j}$-approximations have the following properties $\left(R \subseteq U^{2},\{E, F\} \cup\left\{E_{i}\right\}_{i \in I} \subseteq 2^{U}, j \in J\right)$ :

(1) $\mathscr{R}_{j}^{+}(\varnothing)=\varnothing \subseteq \mathscr{R}_{j}^{-}(\varnothing)$.

(2) $\mathscr{R}_{j}^{+}(U) \subseteq U=\mathscr{R}_{j}^{-}(U)$. 
(3) $\mathscr{R}_{j}^{-}\left(\cap_{i \in I} E_{i}\right)=\cap_{i \in I} \mathscr{R}_{j}^{-}\left(E_{i}\right)$ and $\mathscr{R}_{j}^{+}\left(\cap_{i \in I} E_{i}\right)=$ $\cap_{i \in I} \mathscr{R}_{j}^{+}\left(E_{i}\right)$. Particularly, $\mathscr{R}_{j}^{-}(E) \subseteq \mathscr{R}_{j}^{-}(F)$ and $\mathscr{R}_{j}^{+}(E) \subseteq \mathscr{R}_{j}^{+}(F)$ if $E \subseteq F$.

(4) $\mathscr{R}_{j}^{-}(E)=\left[\mathscr{R}_{j}^{+}\left(E^{c}\right)\right]^{c}$ and $\mathscr{R}_{j}^{+}(E)=\left[\mathscr{R}_{j}^{-}\left(E^{c}\right)\right]^{c}$.

(5) In general, $\mathscr{R}_{j}^{-}\left[\mathscr{R}_{j}^{-}(E)\right] \neq \mathscr{R}_{j}^{-}(E) \quad$ and $\mathscr{R}_{j}^{+}\left[\mathscr{R}_{j}^{+}(E)\right] \neq \mathscr{R}_{j}^{+}(E)$.

Proof. We only prove (3) and (4).

(3) Step 1: obviously, $\mathscr{R}_{j}^{-}\left(\cap_{i \in I} E_{i}\right) \subseteq\left(\cap_{i \in I} \mathscr{R}_{j}^{-}\right)\left(E_{i}\right)$. Conversely, for each $x \in \cap_{i \in I} \mathscr{R}_{j}^{-}\left(E_{i}\right)$ (i.e., $x \in \mathscr{R}_{j}^{-}\left(E_{i}\right)$ for each $i \in I)$, we have $E_{j}(x) \subseteq E_{i}(\forall i \in I)$, and thus, $E_{j}(x) \subseteq \cap_{i \in I} E_{i}$. Hence, $x \in \mathscr{R}_{j}^{-}\left(\cap_{i \in I} E_{i}\right)$, as required.

Step 2: obviously, $\cup_{i \in I} \mathscr{R}_{j}^{+}\left(E_{i}\right) \subseteq \mathscr{R}_{j}^{+}\left(\cup_{i \in I} E_{i}\right)$. Conversely, for each $x \in \mathscr{R}_{j}^{+}\left(\cup_{i \in I} E_{i}\right)$, we have $E_{j}(x) \cap\left(\cup_{i \in I} E_{i}\right) \neq \varnothing$, i.e., $E_{j}(x) \cap E_{i_{0}} \neq \varnothing$ for some $i_{0} \in I$. Thus, $x \in \mathscr{R}_{j}^{+}\left(E_{i_{0}}\right) \subseteq \cup_{i \in I} \mathscr{R}_{j}^{+}\left(E_{i}\right)$, as required.

(4) $x \in \mathscr{R}_{j}^{-}\left(E^{c}\right) \Leftrightarrow E_{j}(x) \subseteq E^{c} \Leftrightarrow E_{j} \quad(x) \cap E=\varnothing \Leftrightarrow x \notin$ $\mathscr{R}_{j}^{+}(E) \Leftrightarrow x \in\left[\mathscr{R}_{j}^{+}(E)\right]^{c}$. The second equality immediately comes by putting $E=F^{c}$ in the first.

Example 2. For $j$-NS $\left(U, R, \lambda_{j}\right)$ in Example 1 , the $\mathscr{R}_{j}$-lower approximations and $\mathscr{R}_{j}$-upper approximations $(j \in\{r, l, i, u\})$, the $\mathscr{R}_{i}$-accuracy measure, the lower approximations $X_{-}$and upper approximations $X_{+}$in the sense of [6] for $P_{\langle r\rangle}$, and the lower approximations $X^{-}$and upper approximations $X^{+}$in the sense of [10] for $N_{r}$ are given in Table 2 (where $Y=\{v, w, y\}$ and $Z=\{v, x, y\}$ ). From Table 2, we can see the approximations in this paper and those in [6] (resp., [10]) are incomparable.

\section{Rough Approximations Induced by E-Topologies}

We will construct rough approximations using $E$-neighborhoods indirectly in this section. Let $\left(U, R, \lambda_{j}\right)$ be $j$-NS $(j \in J)$. We first employ $E_{j}$-neighborhoods to generate a topology $\mathscr{F}_{R, j}$ (called an $E_{j}$-topology) and then call the interior $\mathscr{F}_{R, j}^{-}(X)$ and the closure $\mathscr{F}_{R, j}^{+}(X)$ of a subset $X \subseteq U$ the $\mathscr{J}_{R, j}$-lower approximation and $\mathscr{J}_{R, j}$-upper approximation of $X$, respectively. These kinds of approximations are compared with those in Section 3.

\section{Theorem 3}

(1) $\mathscr{J}_{R, j}=\left\{A \in 2^{U}: E_{j}(x) \subseteq A(\forall x \in A)\right\}$ is a topology on $U$ satisfying $U-A \in \mathscr{J}_{R, j}$ whenever $A \in \mathscr{J}_{R, j}(j \in J)$

(2) Both $\mathscr{J}_{R, N, j}=\left\{A \in 2^{U}: N_{j}(x) \subseteq A(\forall x \in A)\right\}$ and $\mathcal{F}_{R, P, j}=\left\{A \in 2^{U}: P_{j}(x) \subseteq A(\forall x \in A)\right\}$ are topologies on $U(j \in J)$

(3) $\mathscr{J}_{R, u} \subseteq \mathscr{J}_{R, r} \cap \mathscr{J}_{R, l} \subseteq \mathscr{J}_{R, r} \cup \mathscr{J}_{R, l} \subseteq \mathscr{J}_{R, i}$

(4) $\mathscr{F}_{R,\langle u\rangle} \subseteq \mathscr{F}_{R,\langle r\rangle} \cap \mathscr{F}_{R,\langle l\rangle} \subseteq \mathscr{F}_{R,\langle l\rangle} \cup \mathscr{F}_{R,\langle l\rangle} \subseteq \mathscr{F}_{R,\langle i\rangle}$

(5) If $R$ is reflexive, then $\mathscr{J}_{R, P, j} \subseteq \mathscr{J}_{R, j}(j \in J)$

(6) If $R$ is serial, then $\mathscr{J}_{R, P, j} \subseteq \mathscr{J}_{R, j}(j \in\{r, l, u, i\})$
(7) If $R$ is a equivalence relation, then $\mathscr{F}_{R, j}$ is constant for all $j \in J$

Proof. We only prove (1). Obviously, $\mathscr{J}_{R, j}$ is a topology on $U$. For each $A \in \mathscr{J}_{R, j}$ and each $x \in U-A$, we need to prove $E_{j}(x) \subseteq U-A$. Without loss of generality, we assume $E_{j}(x) \neq \varnothing$. Suppose $v \in E_{j}(x) \cap A$. Then, $x \in E_{j}(v)$ (by Theorem 1 (3)) and $E_{j}(v) \subseteq A$ (as $A \in \mathscr{J}_{R, j}$ ), and thus, $x \in A$. This is a contradiction. Therefore, $E_{j}(x) \cap A=\varnothing$, i.e., $E_{j}(x) \subseteq U-A$.

Definition 7. Let $X \subseteq U$ and $j \in J$. Then, $\mathscr{F}_{R, j}^{-}(X)=X^{o}$ (the interior of $X$ in $\left.\left(U, \mathscr{F}_{R, j}\right)\right)$ and $\mathscr{F}_{R, j}^{+}(X)=X^{-}$(the closure of $X$ in $\left.\left(U, \mathscr{f}_{R, j}\right)\right)$ are called the $\mathscr{F}_{R, j}$-lower approximation and $\mathscr{J}_{R, j}$-upper approximation, respectively; $\mathscr{J}_{R, j}^{+}(X)-\mathscr{J}_{R, j}^{-}$ $(X), \mathscr{J}_{R, j}^{+}(X)$, and $U-\mathscr{J}_{R, j}^{+}(X)$ are called $\mathscr{J}_{R, j}$-boundary, $\mathscr{J}_{R, j}$-positive, and $\mathscr{J}_{R, j}$-negative regions of $X$, respectively; $\mathscr{M}_{J, j}(X)=\left(\left|\mathscr{F}_{R, j}^{-}(X)\right| /\left|\mathscr{F}_{R, j}^{+}(X)\right|\right)$ is called the $\mathscr{J}_{R, j}$-accuracy measure of $X$ (if $\left.0<\left|\mathscr{F}_{R, j}^{+}(X)\right|<\aleph_{0}\right)$.

The relation between $\mathscr{M}_{J, j}(X)$ and $\mathscr{M}_{j}(X)(j \in J)$ is given by the following.

Theorem 4. $\mathscr{M}_{J, j}(X) \leq \mathscr{M}_{j}(X)$ for each $j \in J \quad$ (if $\left.0<\left|\mathscr{F}_{R, j}^{+}(X)\right|<\aleph_{0}\right)$.

Proof

(i) Step 1: for each $z \in \mathscr{F}_{R, j}^{-}(X)$, we have $z \in \mathscr{R}_{j}^{-}(X)$ (because $\mathscr{J}_{R, j}^{-}(X)=X^{o}$ and $\left.E_{j}(z) \subseteq X^{o} \subseteq X\right)$, and thus, $\mathscr{J}_{R, j}^{-}(X) \subseteq \mathscr{R}_{j}^{-}(X) \cap X$, which implies

$$
\left|\mathscr{F}_{R, j}^{-}(X)\right| \leq\left|\mathscr{R}_{j}^{-}(X) \cap X\right| .
$$

(ii) Step 2: let $z \in \mathscr{R}_{j}^{+}(X) \cup X$. If $z \in X$, then $z \in \mathscr{J}_{R, j}^{-}(X)$. If $z \notin X$, then $z \in \mathscr{R}_{j}^{+}(X)$, and thus, $E_{j}(z) \cap X \neq \varnothing$. This means there exists $v \in U-\{z\}$ such that $v \in E_{j}(z)$ and $v \in X$. Consequently, for any $V \in \mathscr{J}_{R, j}$ containing $z$, we have $v \in V$. Therefore, $V \cap X \neq \varnothing$, and thus, $z \in \mathscr{F}_{R, j}^{+}(X)$ (because $\left.\mathscr{F}_{R, j}^{+}(X)=X^{-}\right)$. It follows that $\mathscr{R}_{j}^{+}(X) \cup X \subseteq \mathscr{F}_{R, j}^{+}(X)$, and thus,

$$
\frac{1}{\left|\mathscr{F}_{R, j}^{+}(X)\right|} \leq \frac{1}{\left|\mathscr{R}_{j}^{+}(X) \cup X\right|}
$$

From (1) and (2), we can see that $\mathscr{M}_{J, j}(X)=\left(\mid \mathscr{F}_{R, j}^{-}\right.$ $\left.(X)|/| \mathscr{F}_{R, j}^{+}(X) \mid\right) \leq\left(\left|\mathscr{R}_{j}^{-}(X) \cap X\right| /\left|\mathscr{R}_{j}^{+}(X) \cup X\right|\right)=\mathscr{M}_{j}(X)$ $(j \in J)$.

\section{Example 3}

(1) Now, we exemplify an application of rough approximations introduced in this paper. Let $X=\left\{x_{1}, x_{2}, \ldots, x_{50}\right\}$ be a group of people who have just reached Xian Yang Airport (but are not allowed to outbound station) from two countries (by two planes involving 200 people denoted by a set 
TABLE 2: $\mathscr{R}_{j}$-lower approximations and $\mathscr{R}_{j}$-upper approximations $(j \in\{r, l, i, u\})$.

\begin{tabular}{lccccccccccccc}
\hline$X$ & $\mathscr{R}_{u}^{-}(X)$ & $\mathscr{R}_{u}^{+}(X)$ & $\mathscr{R}_{r}^{-}(X)$ & $\mathscr{R}_{r}^{+}(X)$ & $\mathscr{R}_{l}^{-}(X)$ & $\mathscr{R}_{l}^{+}(X)$ & $\mathscr{R}_{i}^{-}(X)$ & $\mathscr{R}_{i}^{+}(X)$ & $\mathscr{M}_{i}(X)$ & $X_{-}$ & $X_{+}$ & $X^{-}$ & $X^{+}$ \\
\hline$\{v\}$ & $\varnothing$ & $U$ & $\{x\}$ & $Y$ & $\varnothing$ & $Z$ & $\{x\}$ & $\{v, y\}$ & 0 & $\varnothing$ & $\{v, x\}$ & $\{x\}$ & $\{v\}$ \\
$\{w\}$ & $\varnothing$ & $Y$ & $\{x\}$ & $Y$ & $\varnothing$ & $\{w, y\}$ & $\{x\}$ & $\{w, y\}$ & 0 & $\{w\}$ & $\{w\}$ & $\{x\}$ & $\{y\}$ \\
$\{x\}$ & $\varnothing$ & $Z$ & $\{x\}$ & $\varnothing$ & $\varnothing$ & $Z$ & $\{x\}$ & $\varnothing$ & 1 & $\varnothing$ & $\{v, x\}$ & $\{x\}$ & $\{v\}$ \\
$\{y\}$ & $\varnothing$ & $U$ & $\{x\}$ & $Y$ & $\varnothing$ & $U$ & $\{x\}$ & $Y$ & 0 & $\{y\}$ & $\{y\}$ & $\{w, x\}$ & $Y$ \\
$\{v, w\}$ & $\varnothing$ & $U$ & $\{x\}$ & $Y$ & $\varnothing$ & $U$ & $\{v, x\}$ & $Y$ & $1 / 3$ & $\{w\}$ & $\{v, w, x\}$ & $\{x\}$ & $\{v, y\}$ \\
$\{v, x\}$ & $\varnothing$ & $U$ & $\{x\}$ & $Y$ & $\varnothing$ & $Y$ & $\{x\}$ & $\{v, y\}$ & $1 / 3$ & $\{v, x\}$ & $\{v, x\}$ & $\{x\}$ & $\{v\}$ \\
$\{v, y\}$ & $\varnothing$ & $U$ & $\{x\}$ & $Y$ & $\varnothing$ & $U$ & $\{v, x\}$ & $Y$ & $1 / 3$ & $\{y\}$ & $Z$ & $\{w, x\}$ & $Y$ \\
$\{w, x\}$ & $\varnothing$ & $U$ & $\{x\}$ & $Y$ & $\varnothing$ & $U$ & $\{x\}$ & $\{w, y\}$ & 1 & $\{w\}$ & $\{v, w, x\}$ & $\{x\}$ & $\{v, y\}$ \\
$\{w, y\}$ & $\varnothing$ & $U$ & $\{x\}$ & $Y$ & $\{w\}$ & $U$ & $\{w, x\}$ & $Y$ & $1 / 3$ & $\{w, y\}$ & $\{w, y\}$ & $\{w, x, y\}$ & $Y$ \\
$\{x, y\}$ & $\varnothing$ & $U$ & $\{x\}$ & $Y$ & $\varnothing$ & $U$ & $\{x\}$ & $Y$ & $1 / 4$ & $\{y\}$ & $Z$ & $\{w, x\}$ & $Y$ \\
$\{v, w, x\}$ & $\varnothing$ & $U$ & $\{x\}$ & $Y$ & $\varnothing$ & $U$ & $\{x\}$ & $Y$ & $1 / 4$ & $\{v, w, x\}$ & $\{v, w, x\}$ & $\{x\}$ & $\{v, y\}$ \\
$Y$ & $Y$ & $U$ & $U$ & $Y$ & $\{w\}$ & $U$ & $U$ & $Y$ & 1 & $\{w, y\}$ & $U$ & $\{w, x, y\}$ & $Y$ \\
$Z$ & $Z$ & $U$ & $\{x\}$ & $Y$ & $\{v, x\}$ & $U$ & $\{v, x\}$ & $Y$ & 1 & $Z$ & $Z$ & $\{v, w, x\}$ & $Y$ \\
$\{w, x, y\}$ & $\varnothing$ & $U$ & $\{x\}$ & $Y$ & $\{w\}$ & $U$ & $\{w, x\}$ & $Y$ & $2 / 3$ & $\{w, y\}$ & $U$ & $\{w, x, y\}$ & $Y$ \\
$U$ & $U$ & $U$ & $U$ & $Y$ & $U$ & $U$ & $U$ & $Y$ & 1 & $U$ & $U$ & $U$ & $Y$ \\
$\varnothing$ & $\varnothing$ & $\varnothing$ & $\{x\}$ & $\varnothing$ & $\varnothing$ & $\varnothing$ & $\{x\}$ & $\varnothing$ & & & & & \\
\hline
\end{tabular}

TABLE 3: $\mathscr{J}_{R, j}$-lower approximations and $\mathscr{J}_{R, j}$-upper approximations $(j \in\{r, l, i, u\})$.

\begin{tabular}{lcccccccccc}
\hline$X$ & $\mathcal{F}_{R, u}^{-}(X)$ & $\mathcal{F}_{R, u}^{+}(X)$ & $\mathcal{F}_{R, r}^{-}(X)$ & $\mathscr{F}_{R, r}^{+}(X)$ & $\mathscr{F}_{R, l}^{-}(X)$ & $\mathcal{F}_{R, l}^{+}(X)$ & $\mathscr{F}_{R, i}^{-}(X)$ & $\mathcal{F}_{R, i}^{+}(X)$ & $\mathscr{M}_{J, i}(X)$ & $\mathscr{M}_{i}(X)$ \\
\hline$\{v\}$ & $\varnothing$ & $U$ & $\varnothing$ & $\{v, w, y\}$ & $\varnothing$ & $\varnothing$ & $\varnothing$ & $\{v, w, y\}$ & 0 & 0 \\
$\{w\}$ & $\varnothing$ & $U$ & $\varnothing$ & $\{v, w, y\}$ & $\varnothing$ & $U$ & $\varnothing$ & $\{v, w, y\}$ & 0 & 0 \\
$\{x\}$ & $\varnothing$ & $U$ & $\{x\}$ & $U$ & $\varnothing$ & $U$ & $\{x\}$ & $U$ & $1 / 4$ & 1 \\
$\{y\}$ & $\varnothing$ & $U$ & $\varnothing$ & $\{v, w, y\}$ & $\varnothing$ & $U$ & $\varnothing$ & $\{v, w, y\}$ & 0 & 0 \\
$\{v, w\}$ & $\varnothing$ & $U$ & $\varnothing$ & $\{v, w, y\}$ & $\varnothing$ & $U$ & $\varnothing$ & $\{v, w, y\}$ & 0 & $1 / 3$ \\
$\{v, x\}$ & $\varnothing$ & $U$ & $\{x\}$ & $U$ & $\varnothing$ & $U$ & $\{x\}$ & $U$ & $1 / 4$ & $1 / 3$ \\
$\{v, y\}$ & $\varnothing$ & $U$ & $\varnothing$ & $\{v, w, y\}$ & $\varnothing$ & $U$ & $\varnothing$ & $\{v, w, y\}$ & 0 & $1 / 3$ \\
$\{w, x\}$ & $\varnothing$ & $U$ & $\{x\}$ & $U$ & $\varnothing$ & $U$ & $\{x\}$ & $U$ & $1 / 4$ & 1 \\
$\{w, y\}$ & $\varnothing$ & $U$ & $\varnothing$ & $\{v, w, y\}$ & $\varnothing$ & $U$ & $\varnothing$ & $\{v, w, y\}$ & 0 & $1 / 3$ \\
$\{x, y\}$ & $\varnothing$ & $U$ & $\{x\}$ & $U$ & $\varnothing$ & $U$ & $\{x\}$ & $U$ & $1 / 4$ & $1 / 4$ \\
$\{v, w, x\}$ & $\varnothing$ & $U$ & $\{x\}$ & $U$ & $\varnothing$ & $U$ & $\{x\}$ & $U$ & $1 / 4$ & $1 / 4$ \\
$\{v, w, y\}$ & $\varnothing$ & $U$ & $\{v, w, y\}$ & $\{v, w, y\}$ & $\varnothing$ & $U$ & $\{v, w, y\}$ & $\{v, w, y\}$ & 1 & 1 \\
$\{v, x, y\}$ & $\varnothing$ & $U$ & $\{x\}$ & $U$ & $\varnothing$ & $U$ & $\{x\}$ & $U$ & $1 / 4$ & 1 \\
$\{w, x, y\}$ & $\varnothing$ & $U$ & $\{x\}$ & $U$ & $\varnothing$ & $U$ & $\{x\}$ & $U$ & $1 / 4$ & $2 / 3$ \\
$U$ & $U$ & $U$ & $U$ & $U$ & $U$ & $U$ & & $U$ & 1 & 1 \\
$\varnothing$ & $\varnothing$ & $\varnothing$ & $\varnothing$ & $\varnothing$ & $\varnothing$ & $\varnothing$ & $\varnothing$ & $\varnothing$ & & \\
\hline
\end{tabular}

$\left.U=\left\{x_{1}, x_{2}, \ldots, x_{200}\right\}\right)$ and will attend a meeting holding in Xi'an. Assume that $x_{1}$ and $x_{2}$ are actually infected of asymptomatic infection of new coronavirus, $E_{j}(x) \in 2^{U}$ consists of $x$ and all $y \in U$ who contacted $x$ after $x$ had contacted one of $x_{1}$ and $x_{2}\left(x \in U-\left\{x_{1}, x_{2}\right\}\right)$, and $E_{j}\left(x_{1}\right)=\left\{x_{1}\right\} \quad$ and $E_{j}\left(x_{2}\right)=\left\{x_{2}\right\}$. To insure the safety of this meeting, $\mathscr{R}_{j}^{-}(X)=\left\{x \in U \mid E_{j}(x) \subseteq X\right\}=\left\{x_{1}, x_{2}\right\} \cup\{x \in X \mid$ each person contacting $x$ directly after $x$ contacts one of $x_{1}$ and $x_{2}$ is in $\left.X\right\}$ can be looked to be the set of all people who must run a nucleic acid test, and $\mathscr{R}_{j}^{+}(X)=X \cup$

$\{x \in U$ $\left.\mid E_{j}(x) \cap X \neq \varnothing\right\}=X \cup\{x \in U-X \mid$ some person contacting $x$ directly after $x$ contacts one of $x_{1}$ and $x_{2}$ is in $X$ \} can be looked to be the set of all people who should run a nucleic acid test.

(2) For $j$-NS $\left(U, R, \lambda_{j}\right)$ in Example 1 , the $\mathscr{J}_{R, j}$-lower approximations and $\mathscr{J}_{R, j}$-upper approximations $(j \in\{r, l, i, u\})$ and the $\mathscr{M}_{J, j}$-accuracy measure are given in Table 3.

\section{Concluding Remarks}

Motivated by topology, this article has initiated two new rough approximations by introducing a new class of neighborhood systems (called $E_{j}$-neighborhoods) using $j$-neighborhoods. We have probed the main features and formulated the concepts of $E_{j}$-lower and $E_{j}$-upper approximations and $E_{j}$-accuracy measure which are had contacted one of induced from different types of $j$ and compared them. We complete this work by studying these concepts from a topological view and comparing them. In all comparisons, we obtain higher accurate approximations in the case of $j=i$.

In the upcoming works, we will study new types of neighborhoods in rough set theory and use them to define a 
topological structure. Also, we will investigate the $E_{j}$-neighborhoods and approximations on the fuzzy rough set motivated from fuzzy control problems and fractional-order nonlinear systems.

\section{Data Availability}

The data used to support the findings of this study are included within the article.

\section{Conflicts of Interest}

The authors declare that there are no conflicts of interest regarding the publication of this paper.

\section{Acknowledgments}

This work was supported by the National Natural Science Foundation of China under Grant nos. 11771263 and 61967001.

\section{References}

[1] Y. Zhou, H. Liu, J. Cao, and S. Li, “Composite learning fuzzy synchronization for incommensurate fractional-order chaotic systems with time-varying delays," International Journal of Adaptive Control and Signal Processing, vol. 33, no. 12, pp. 1739-1758, 2019.

[2] Y. Zhou, H. Wang, and H. Liu, "Generalized function projective synchronization of incommensurate fractional-order chaotic systems with inputs saturation," International Journal of Fuzzy Systems, vol. 21, no. 3, pp. 823-836, 2019.

[3] Z. Pawlak, "Rough sets," International Journal of Information and Computing Sciences, vol. 11, no. 5, pp. 341-356, 1982.

[4] Z. Pawlak, Rough Sets, Theoretical Aspects of Reasoning about Data, Kluwer Acadmic Publishers, Dordrecht, The Netherlands, 1991.

[5] E. A. Abo-Tabl, "Rough sets and topological spaces based on similarity," International Journal of Machine Learning and Cybernetics, vol. 4, pp. 451-458, 2013.

[6] M. Atef, A. M. Khalil, S.-G. Li, A. Azzam, and A. El Atik, "Comparison of six types of rough approximations based on $j$-neighborhood space and $j$-adhesion neighborhood space," Journal of Intelligent and Fuzzy Systems, vol. 39, no. 3, pp. 4515-4531, 2020.

[7] A. Skowron and J. Stepaniuk, "Tolerance approximation spaces," Fundamenta Informaticae, vol. 27, pp. 245-253, 1996.

[8] R. Slowinski and D. Vanderpooten, "A generalized definition of rough approximations based on similarity," IEEE Transactions on Knowledge and Data Engineering, vol. 12, pp. 331-336, 2000.

[9] S. Sun, L. Li, and K. Hu, "A new approach to rough set based on remote neighborhood systems," Mathematical Problems in Engineering, vol. 2019, Article ID 8712010, 8 pages, 2019.

[10] Y. Y. Yao, "Two views of the theory of rough sets in finite universes," International Journal of Approximate Reasoning, vol. 15, pp. 291-317, 1996.

[11] Y. Y. Yao, "Relational interpretations of neighborhood operators and rough set approximation operators," Information Sciences, vol. 111, pp. 239-259, 1998.

[12] K. Zhang, J. M. Zhan, and W. Z. Wu, "Novel fuzzy rough set models and corresponding applications to multi-criteria decision-making," Fuzzy Sets and Systems, vol. 383, pp. 92-126, 2020.
[13] W. Zhu, "Relationship between generalized rough sets based on binary relation and covering," Information Sciences, vol. 179, pp. 210-225, 2009.

[14] Y. Y. Yao, Generalized Rough Set Models, Rough Sets in Knowledge Discovery 1, L. Polkowski and A. Skowron, Eds., pp. 286-318, Physica Verlag Springer, Berlin, Germany, 1998.

[15] A. A. Allam, M. Y. Bakeir, and E. A. Abo-Tabl, "New approach for basic rough set concepts," in International workshop on rough sets, fuzzy sets, data mining, and granular computing. Lecture Notes in Artificial Intelligence, vol. 3641, pp. 64-73, Springer, Berlin, Germany, 2005.

[16] M. E. Abd El-Monsef, O. A. Embaby, and M. K. El-Bably, "Comparison between rough set approximations based on different topologies," International Journal of Granular Computing, Rough Sets and Intelligent Systems, vol. 3, no. 4, pp. 292-305, 2014.

[17] P. K. Singh and S. Tiwari, "Topological structures in rough set theory: a survey," Hacettepe Journal of Mathematics and Statistics, vol. 49, no. 4, pp. 1270-1294, 2020.

[18] E. A. Abo-Tabl, "On links between rough sets and digital topology," Applied Mathematics, vol. 5, pp. 941-948, 2014.

[19] A. Azzam, A. M. Khalil, and S.-G. Li, "Medical applications via minimal topological structure," Journal of Intelligent and Fuzzy Systems, vol. 39, no. 3, pp. 4723-4730, 2020, (in prepress).

[20] M. Hosny, "On generalization of rough sets by using two different methods," Journal of Intelligent and Fuzzy Systems, vol. 35, no. 1, pp. 979-993, 2018.

[21] E. F. Lashin, A. M. Kozae, A. A. Abo Khadra, and T. Medhat, "Rough set theory for topological spaces," IInternational Journal of Approximate Reasoning, vol. 40, pp. 35-43, 2005.

[22] Z. Li, T. Xie, and Q. Li, "Topological structure of generalized rough sets," Computers \& Mathematics with Applications, vol. 63, pp. 1066-1071, 2012.

[23] A. S. Nawar, M. K. El-Bably, and A. A. El-Atik, "Certain types of coverings based rough sets with application," Journal of Intelligent and Fuzzy Systems, vol. 39, no. 3, pp. 3085-3098, 2020, (in pre-press).

[24] A. S. Salama, "Sequences of topological near open and near closed sets with rough applications," Filomat, vol. 34, no. 1, pp. 51-58, 2020.

[25] Q. E. Wu, T. Wang, Y. X. Huang, and J. S. Li, “Topology theory on rough sets," IEEE Transactions on Systems, Man and Cybernetics, Part B (Cybernetics), vol. 38, no. 1, pp. 68-77, 2008.

[26] Z. Pei, D. Pei, and L. Zheng, "Topology vs generalized rough sets," International Journal of Approximate Reasoning, vol. 52, no. 2, pp. 231-239, 2011. 